\section{Verleiten sie Jugendliche zum späteren Tabakrauchen?}

\author{
Elektronische Zigaretten werden als Nikotinquelle zunehmend \\ populär, v. a. auch unter Jugendlichen. Dies gilt sogar für \\ Teenager, die zuvor noch niemals Tabak geraucht haben. \\ A. M. Leventhal et al. gingen nun der Frage nach, ob der \\ Gebrauch von E-Zigaretten bei Jugendlichen die \\ Wahrscheinlichkeit steigert, später auf Tabak umzusteigen. \\ JAMA 2015; 314: 700-707
}

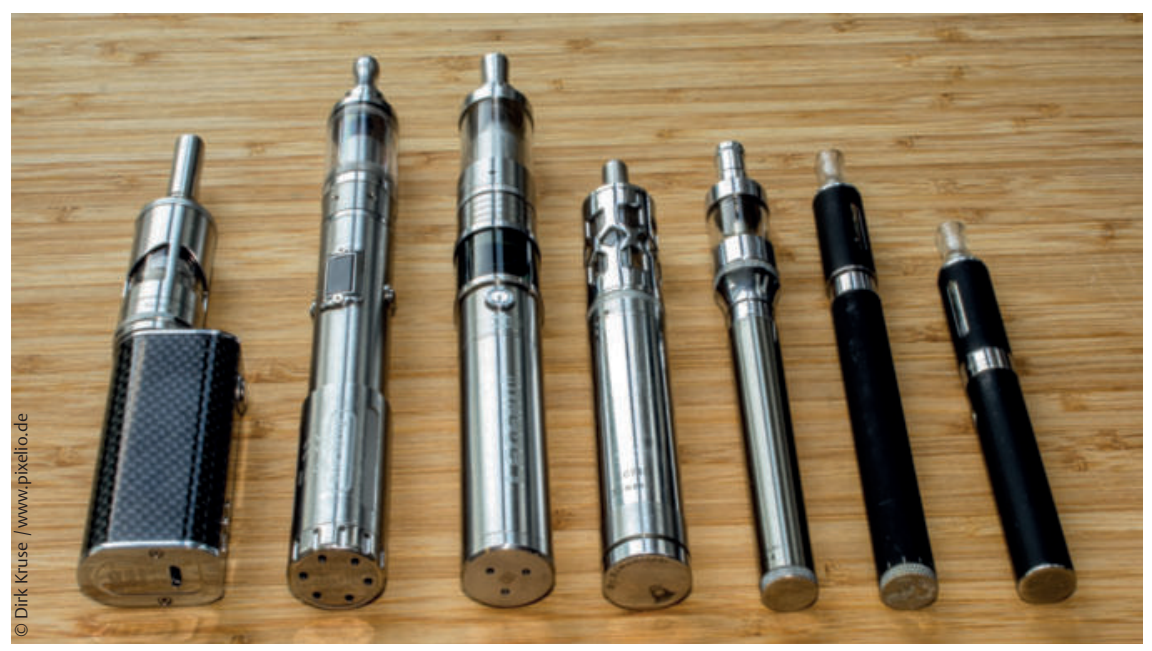

Die Autoren analysierte hierzu longitudinal eine Kohorte von Jugendlichen, die zu Studienbeginn im Herbst 2013 durchschnittlich 14,1 Jahre alt waren und die 9. Klasse einer High School besuchten. Follow-up-Termine fanden nach 6 und nach 12 Monaten statt. Eingang in die Auswertung fanden Schüler, die zu Beginn der Studie angaben, noch niemals Tabak geraucht zu haben und beide Folgetermine absolvierten. Mittels Fragebogen gaben die Teilnehmer eingangs Auskunft, ob sie schon einmal E-Zigaretten benutzt hatten. Bei den Follow-up-Terminen wurden sie u.a. befragt, ob sie innerhalb der vergangenen 6 Monate irgendwelche brennbaren Tabakprodukte, Zigaretten, Zigarren oder Wasserpfeife geraucht hatten sowie nach der Anzahl der verschiedenen Produkte. Erfasst wurden außerdem verschiedene soziodemografische Daten.

Eingang in die Analyse fanden 2530 Schüler, von denen 222 angaben, schon einmal E-Zigaretten benutzt zu haben. Nach 6 Monaten war das Rauchen von Tabak in der Gruppe mit anfänglichem Konsum von E-

\begin{abstract}
Fazit
In der Gruppe der untersuchten Jugendlichen war die Wahrscheinlichkeit für Tabakrauchen im Folgejahre bei denjenigen höher, die eingangs E-Zigaretten konsumiert hatten, so die Autoren.
\end{abstract}

Dr. Johannes Weiß, Bad Kissingen

\section{Erstes internationales Patientenregister}

Die Lungenerkrankung Primary Ciliary Dyskinesia (PCD) verursacht lang anhaltende und immer wiederkehrende Atemwegsinfekte. PCD tritt bereits bei Kindern auf und wird durch einen Gendefekt ausgelöst. Die Flimmerhärchen in den Atemwegen, Nasennebenhöhlen und dem Mittelohr werden unbeweglich. In der Folge sammelt sich Schleim in diesen Organen an, der nicht abtransportiert werden kann. Es entstehen irreversible, sackförmige Bronchienweiterungen, die langfristig auch zu Lungenversagen führen können. Bislang steht keine erfolgreiche Therapie zur Behandlung der Erkrankung zur Verfügung. Trotz wissenschaftlicher Fortschritte in der Erforschung seltener Erkrankungen konnten viele Aspekte wie die unterschiedliche Symptomatik bei Patienten und die Auswirkungen einzelner therapeutischer Interventionen noch nicht vollständig verstanden werden. Zur Generierung grundlegend neuer Erkenntnisse wird eine Vielzahl dokumentierter Fälle benötigt. Patientenregister sind gerade bei seltenen Erkrankungen effektive Instrumente zur Dokumentation der gesundheitlichen Konstitution der betroffenen Patienten, des Krankheitsverlaufs und der Reaktion auf die angewandten Therapien. Im Dezember wurde im European Respiratory Journal eine Studie (DOI: 10.1183/13993003.007762015) veröffentlicht, die beschreibt, wie die Forscher das neue Register als eine internationale Online-Plattform zur systematischen Dokumentation von PCD-Diagnosen, -Symptomen, -Therapien und -Krankheitsverläufen einsetzen .Die Arbeitsgruppe um H. Omran, Münster, kooperierte dazu eng mit international führenden Wissenschaftlern und Patientenorganisationen. Bisher wurden rund 201 Patienten in das PCD-Register aufgenommen. Durch die Rekrutierung weiterer Referenzzentren sollen künftig mehr Patienten einbezogen werden. Zudem sollen regionale Registrierungs-Koordinatoren etabliert werden, um den Forschungsstand breiter zu kommunizieren und den Patientenzugang aus Ländern außerhalb des Konsortiums zu erleichtern.

Nach einer Mitteilung der Westfälischen Wilhelms-Universität Münster 